\title{
Sex Differences in PTSD Symptoms: A Differential Item Functioning Approach
}

\author{
Siobhan Murphy and Ask Elklit \\ University of Southern Denmark
}

\author{
Yoke Yong Chen and Siti Raudzah Ghazali \\ Universiti Malaysia Sarawak
}

\author{
Mark Shevlin \\ Ulster University
}

\begin{abstract}
Objective: Evidence has suggested there are sex differences in posttraumatic stress disorder (PTSD) symptom expression; however, few studies have assessed whether these differences are due to measurement invariance. This study aimed to examine sex differences in PTSD symptoms based on the Diagnostic and Statistical Manual of Mental Disorders (5th ed.; DSM-5) using differential item functioning (DIF). Method: Confirmatory factor analysis was conducted on the DSM-5 model of PTSD, followed by a multiple indicators multiple causes (MIMIC) model to examine possible DIF using the PTSD Checklist for DSM-5. Data were analyzed from a Malaysian adolescent sample $(n=481)$ of which $61.7 \%$ were female, with a mean age of 17.03 years. Results: The results indicated the presence of DIF for 2 of 20 PTSD criteria. Females scored significantly higher on emotional cue reactivity (B4), and males reported significantly higher rates of reckless or self-destructive behavior (E2) while statistically controlling for the latent variables in the model. However, the magnitude of these item-level differences was small. Conclusion: These findings indicate that despite the presence of DIF for 2 DSM-5 symptoms, this does not provide firm support for nonequivalence across sex.
\end{abstract}

Clinical Impact Statement

This study suggests that although sex differences were observed in 2 out of 20 posttraumatic stress disorder (PTSD) symptom criteria, the magnitude of these effects was small and may be the result of gender role stereotypes rather than a bias in diagnostic criteria. Given the paucity of studies examining sex differences in PTSD symptom expression based on the Diagnostic and Statistical Manual of Mental Disorders (5th ed.), more research is needed across different cultures and types of traumatic exposure before firm conclusions can be made.

Keywords: posttraumatic stress disorder, DSM-5, differential item functioning, gender differences, PTSD Checklist for $D S M-5$

Supplemental materials: http://dx.doi.org/10.1037/tra0000355.supp

The Diagnostic and Statistical Manual of Mental Disorders (DSM-5: American Psychiatric Association, 2013) diagnosis of posttraumatic stress disorder (PTSD) has undergone significant changes in nosology, definition of a traumatic stressor, and the number and nature of symptom criteria (Weathers, 2017). No-

Siobhan Murphy and Ask Elklit, National Centre of Psychotraumatology, University of Southern Denmark; Yoke Yong Chen and Siti Raudzah Ghazali, Faculty of Medicine and Health Science, Universiti Malaysia Sarawak; Mark Shevlin, Psychology Research Institute, Ulster University.

Funding for this study was provided to Siti Raudzah Ghazali by Malaysian Ministry of Higher Education Grant FRGS/SS02/(01)/1144/2014 (11).

Correspondence concerning this article should be addressed to Siobhan Murphy, National Centre of Psychotraumatology, Department of Psychology, University of Southern Denmark, Campusvej 55, Odense M, Denmark 230. E-mail: smurphy@health.sdu.dk tably, the tripartite model was replaced with a four-factor model based on a large body of evidence from confirmatory factor analytic findings demonstrating that PTSD is better comprised of four rather than three dimensions (Yufik \& Simms, 2010). The four symptom clusters are intrusions (Criterion B), avoidance (Criterion $\mathrm{C}$ ), negative alterations in cognitions and mood (NACM; Criterion D), and alterations in arousal and reactivity (Criterion E). Additional modifications are reflected in the separation of the DSM-IV (American Psychiatric Association, 1994) Criterion $C$ of active avoidance and emotional numbing into two separate clusters and the addition of three symptoms (blame, persistent negative emotions, and reckless or selfdestructive behavior).

Support for the DSM-5 four-factor model of PTSD has been evidenced from confirmatory factor analytic findings using different measures and across numerous trauma and community samples and cultures (Biehn et al., 2013; Contractor et al., 2013; Elhai et al., 2012; Tay, Jayasuriya, Jayasuriya, \& Silove, 2017). More 